\title{
Failure investigation as a route to improving integrity of titanium alloys in service
}

\author{
C. Collins ${ }^{a, *}$, F.F. Dear ${ }^{b}$, D. Rugg ${ }^{a}$, D. Dye ${ }^{b}$ \\ ${ }^{\mathrm{a}}$ Rolls-Royce plc, Derby, UK; ${ }^{\mathrm{b}}$ Imperial College London, London, UK. \\ *christopher.collins3@rolls-royce.com
}

Increasing demands on titanium alloys in aerospace applications have driven a push towards deeper understanding of their behaviour in service. This extends from component performance during planned operation to damage mechanisms and how parts may ultimately fail. Investigation of damage and failure requires a comprehensive framework of techniques in order to identify a root cause, and further the understanding of failure mechanisms. It is crucial to defining and improving component lifetimes via a design optimisation feedback loop. This paper presents an overview of the techniques used in state-of-the-art industrial titanium alloy failure investigation, highlighting the inherent reciprocal links to frontline research and the need for constant innovation.

\section{Introduction}

This review surveys the usage of several techniques, divided into core, specialist and future categories according to their levels of industrial access and readiness. Good understanding of when and how best to apply these techniques is key to unlocking the complexities of in-service component failures. Core techniques available in-house are used in both routine and highly detailed investigations, and form a broad basis from which externally supplied specialist or novel techniques can be employed as required. A number of factors determine the applicability of each technique to different types of investigation, Fig. 1, and these have been used to divide up the categories in this paper.

(a)

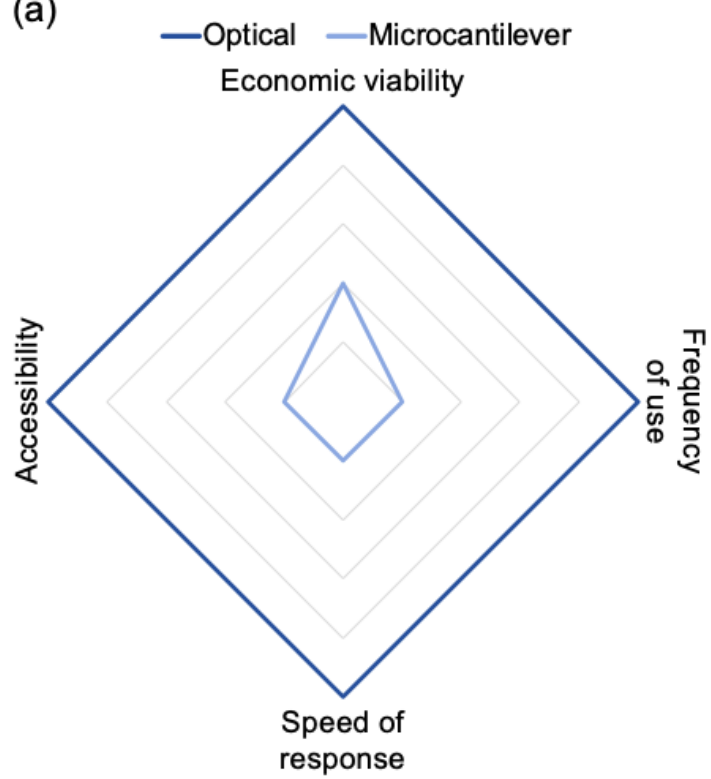

(b)

\section{-FIB-SIMS —DSIMS \\ Economic viability}

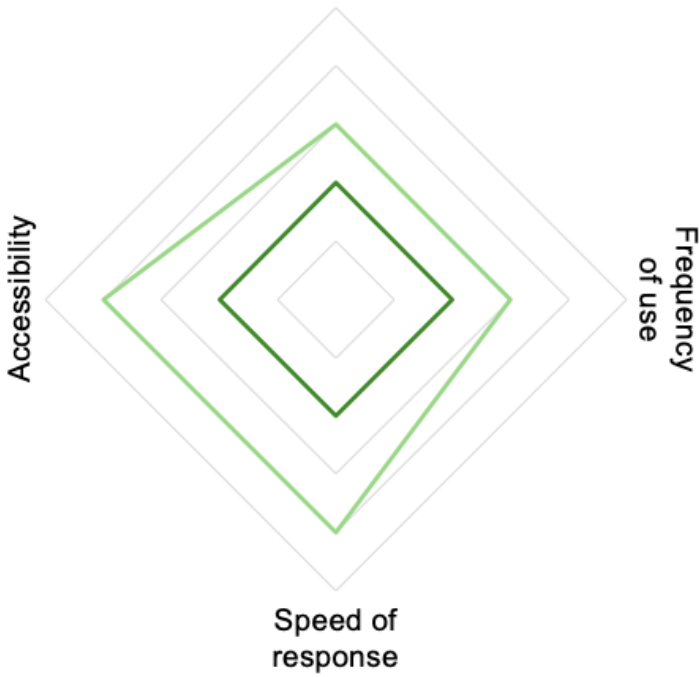

Figure 1: Several factors determine the usage of techniques in failure investigation, schematically shown here in radar plots. (a) Optical microscopy provides a rapid overview of samples and is used in every investigation, while microcantilever testing of local mechanical properties is still under development as an investigative tool. (b) FIB-SIMS is commonly applied in academic research, but DSIMS results are more accessible for industry.

\section{Core techniques}

Many of the techniques used routinely in industrial failure investigation have been available for decades but remain essential. Among these are high-quality macro photography, stereomicroscopy and secondary electron imaging in scanning electron microscopy (SEM). Recent refinements and advances in hardware and software increase the depth of insight provided while also allowing for faster response times, which can be crucial in supporting root cause understanding in the hours and days immediately following an in-service event. This section explores some of these.

\section{Automated 3D optical microscopy}

Optical microscopy is the first port of call for many investigations and has benefitted greatly from the development of automated focus stack imaging. Quantitative topographical information can be extracted from the stitched image dataset, allowing 3D representations of the region of interest to be made, as well as allowing features such as surface relief depths or fracture surface inclinations to be measured, Fig. 2 [1]. These systems allow such data to be recorded at coarser length scales and for larger areas than are feasibly observed in analogous SEM systems. 

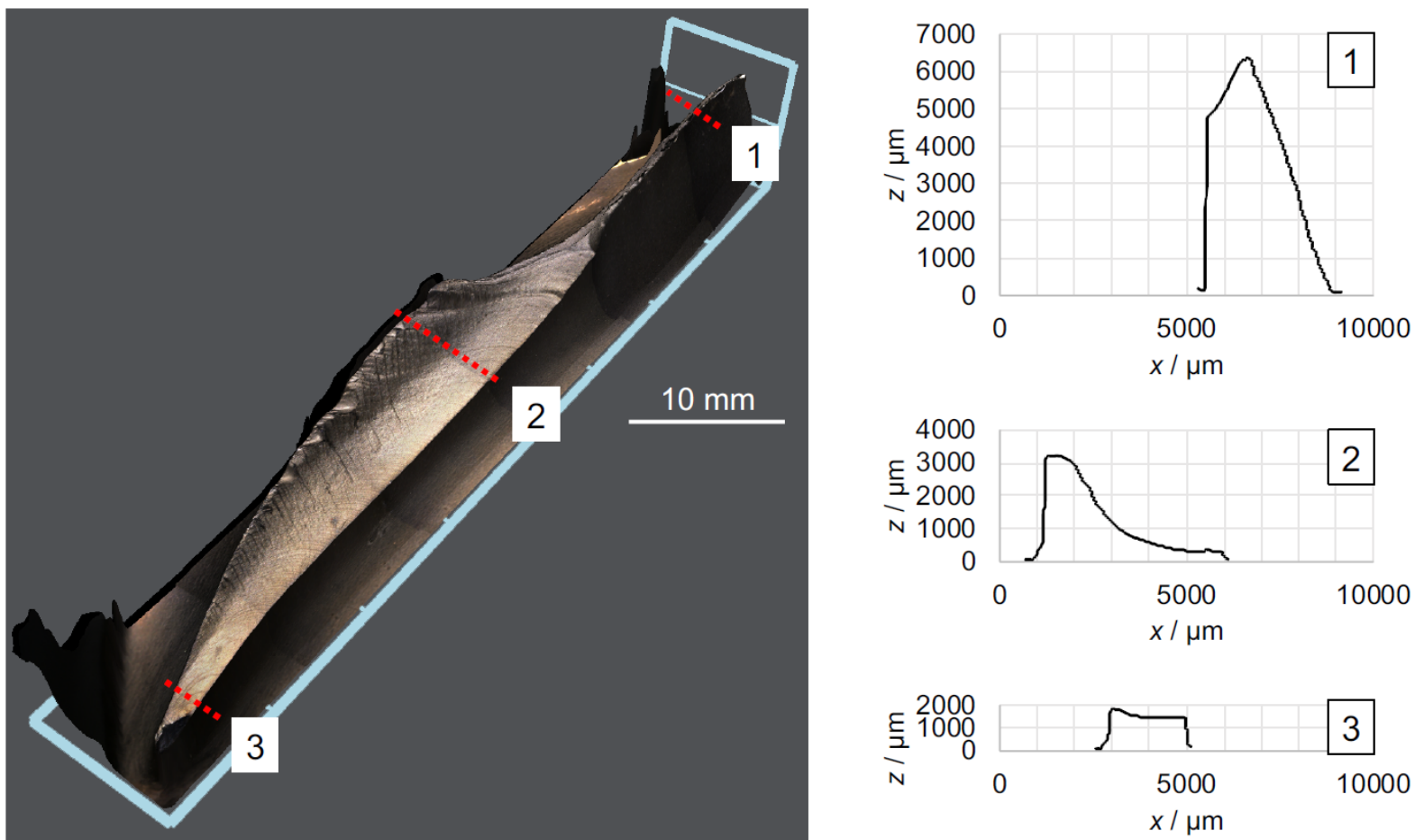

Figure 2: A 3D optical micrograph of a high cycle fatigue fracture in a compressor blade aerofoil, captured with a Zeiss SmartZoom 5 system. Optical images are recorded in a stitched focus stack, calibrated so that accurate topographical information can be extracted, as demonstrated in the three height profiles shown.

Automated SEM image collation and capture

Recent SEM software advances [2] have enabled a step change in SEM usage for the analysis of fracture surfaces. Using the metadata for each image to locate it in space, a 3D collation of all images in a dataset can be produced automatically to give much better and faster contextualisation of feature locations, Fig. 3(a). This contextualisation also enables data input for cross-checking of stress modelling by providing quantitative data regarding the transition from fatigue to fast fracture. Comparison to earlier optical examinations is also possible with software capable of calibrating stage coordinates to an optical micrograph or macro photograph.

Another software development of great advantage to industrial SEM users is automatic capture of images across a large ( $x, y$ ) area with automatic focussing on each tile of a stitched image, Fig. 3(b). This allows SEMs to be used around the clock without the need for an investigator to be present, freeing up time for analysis and improving the cost effectiveness of the equipment. This type of software also allows imaging of very large areas, along with easy contextualisation of features.

(a)

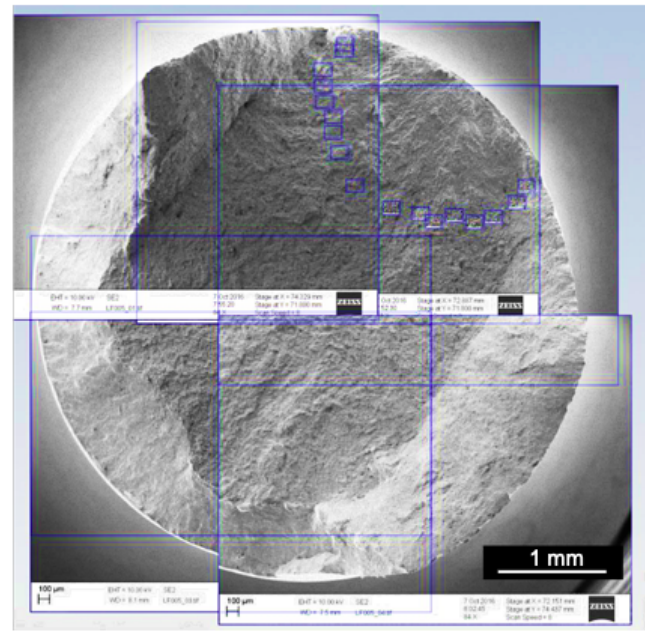

(b)

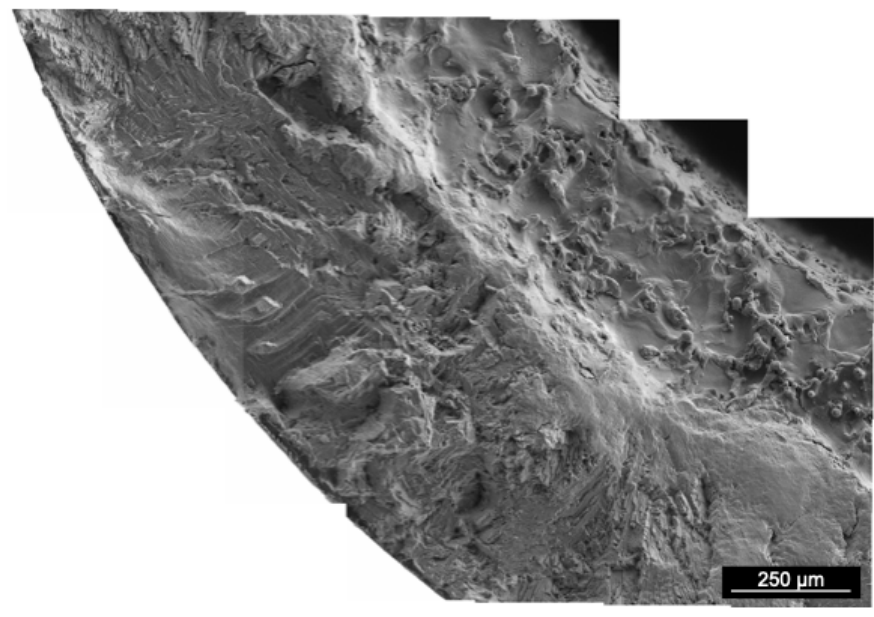

Figure 3: (a) An example of image collation in Zeiss's SmartBrowse software, showing SEM images located relative to one another using metadata. Here, the boundary between regions of different fracture behaviour was clearly identified in the context of the fracture surface as a whole. (b) A stitched image of a fracture surface captured using Zeiss's SmartStitch software, providing a large field of view while retaining good resolution.

\section{EBSD fractography}

While a standard technique on flat, polished specimens, the use of EBSD on fracture surfaces is less well established and presents a range of challenges [3]. Surface roughness is the first barrier to using this technique on fracture surfaces, but it is possible to successfully index patterns on low- $\Delta K$ fatigue facets and other relatively flat features, Fig. 4. Inclination of the fracture surface relative to part geometry is often a complicating factor. Low indexing rates mean that care must be taken in interpreting results. It is anticipated that the development of CMOS detectors will allow for improvements in this technique. 

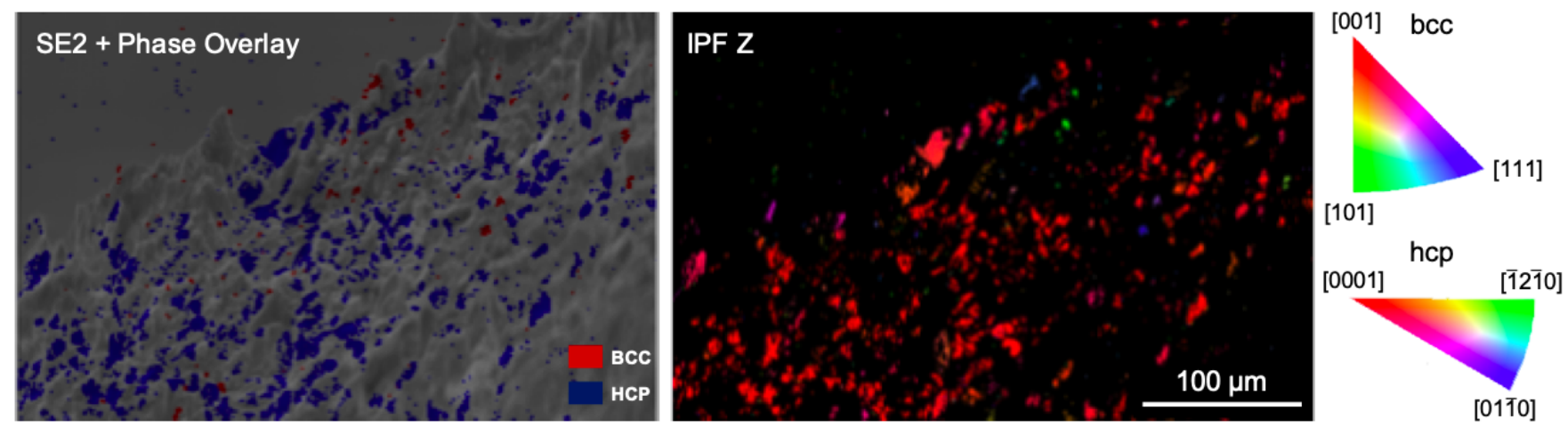

Figure 4: EBSD fractography of a facetted fracture surface, demonstrating the capabilities and limitations of current systems. Although several facets have been successfully indexed, the surface roughness of the fracture prevents complete mapping and remains challenging.

\section{Specialist techniques}

A range of more specialist techniques has become part of the arsenal of Rolls-Royce failure investigators, used to complement our core techniques where greater complexity is encountered during an investigation. These techniques are typically provided by academic institutions or specialist contract companies with which Rolls-Royce maintains close working relationships. This expands the toolkit of investigators beyond what is feasibly performed in-house, allowing access to highly specific expertise in cases where it is required only rarely.

Focussed ion beam secondary ion mass spectrometry (FIB-SIMS)

In cases where SEM-EDX is unable to chemically or spatially resolve features under investigation, for instance where light elements are of interest, FIB-SIMS may be used. A FIB is used to sputter ions from a specified area, which are then measured in a mass spectrometer to identify their chemical species. This chemical analysis can be performed at very shallow depths [4], to analyse surface layers, or to construct coarse depth profiling of a cross-section, Fig. 5(a).

Dynamic secondary ion mass spectrometry (DSIMS)

DSIMS involves similar methods to FIB-SIMS, but measures bulk composition by targeting a larger area with FIB sputtering. This can sample at depths up to $30 \mu \mathrm{m}$ for typical industrial alloys, and can be used in either a relative sense, comparing numbers of counts, or an absolute sense, when calibrated against a standard specimen. Measurement of oxygen, carbon and nitrogen in titanium alloys is possible, Fig. 5(b), and Rolls-Royce has been active in driving this technique forward to achieve repeatability in measurement of these species at the $10 \mathrm{ppm}$ level in calibrated materials.

(a)

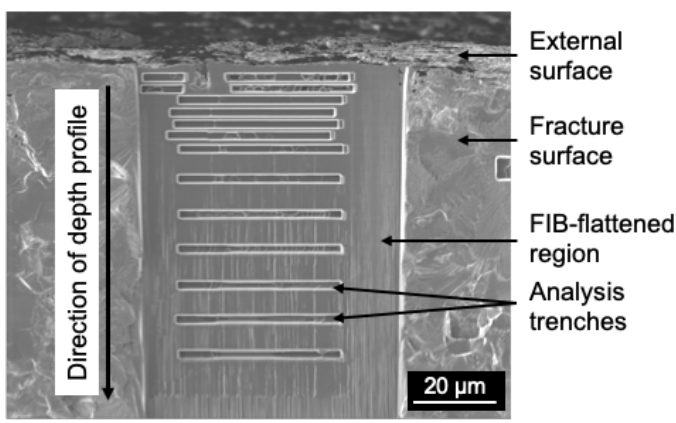

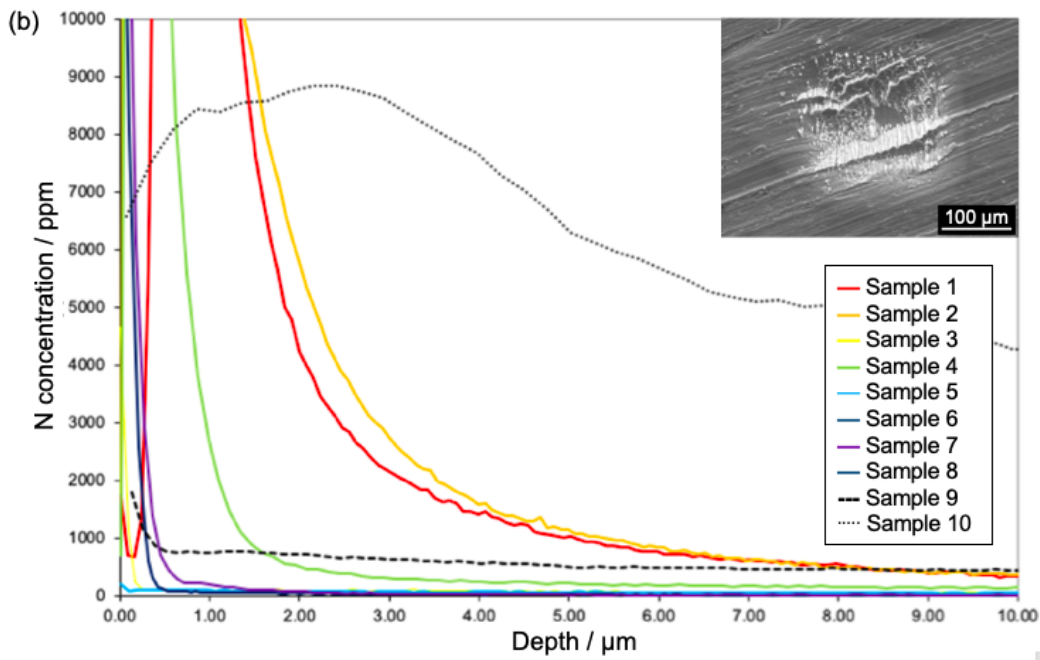

Figure 5: Two SIMS techniques are applicable to failure investigation. (a) An SEM image shows FIB-SIMS trenches made to create a coarse depth profile across a fracture surface, requiring prior flattening with glancing-angle FIB milling for comparable measurements. (b) DSIMS provides finer-scale depth profiling of nitrogen concentration in ten different Ti$6 \mathrm{Al}-4 \mathrm{~V}$ (wt.\%) surfaces, and is made fully quantitative by calibration to a standard. Inset is an SEM micrograph showing a sputtered crater after measurement.

Widefield confocal microscopy

Confocal microscopy is another recent addition to the failure investigation toolkit, allowing quantitative topographical examination of as-machined surfaces or fracture surfaces [5]. In the observation of fractures, it provides a useful route to understanding crack path behaviour, Fig. 6 . 
(a)

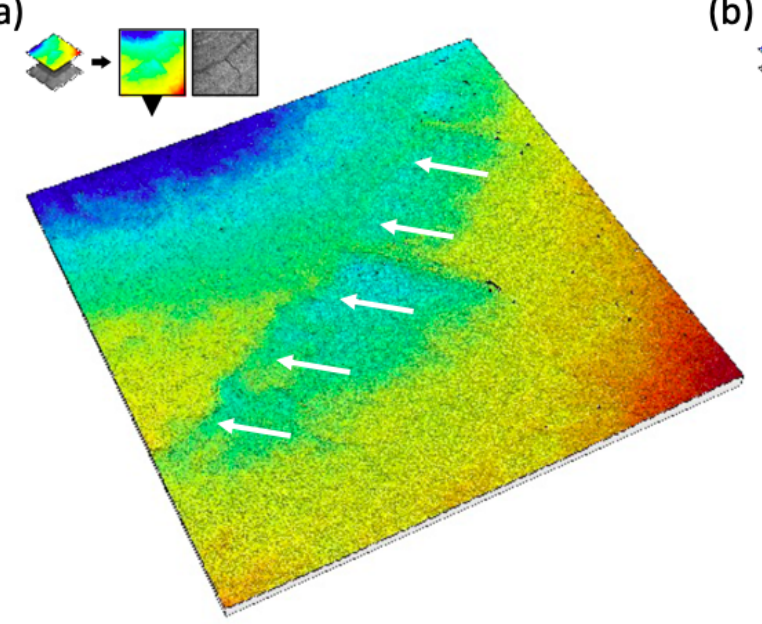

(b)

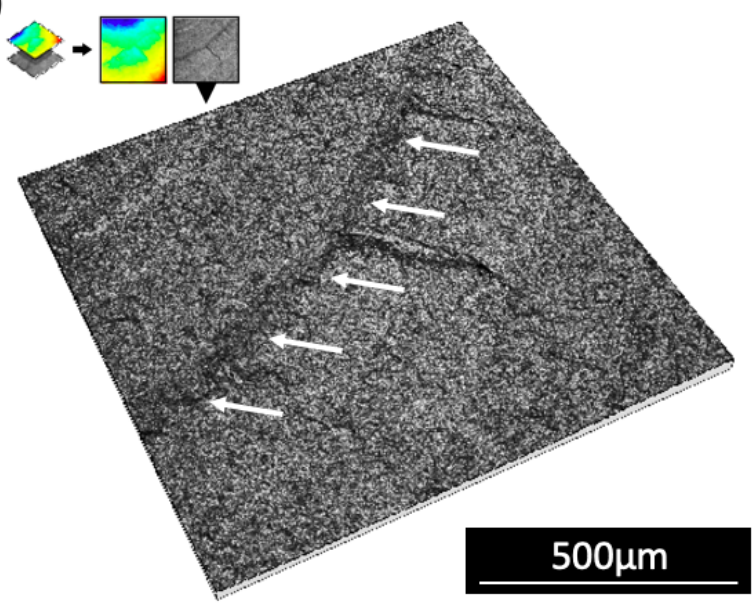

Figure 6: Widefield confocal microscopy analysis of a fracture surface on a compressor blade, acquired using a Zeiss SmartProof 5 system to produce a height map (a) and an optical image (b). The arc-like feature indicated in the images is a beach-mark resulting from high cycle fatigue.

Spatially resolved acoustic spectroscopy (SRAS)

For instances where texture information is required across large areas, EBSD may be too slow for industrial response times, so that the development of SRAS provides a useful alternative [6]. Laser-induced acoustic pulses are sent through the sample, and the acoustic velocity response is measured, which depends on crystallographically-determined acoustic impedance. Although it does not quantify specific grain orientations, the resulting velocity map gives an indication of grain size and texture intensity, Fig. 7. As with EBSD, the surface needs to be prepared to a high polish finish, but larger samples may be inspected.

\section{FIB lift-outs}

Local structure and chemistry at the sub-micron scale may be of interest for more in-depth investigations, making FIB lift-out of a feature for subsequent transmission electron microscopy (TEM) useful. A dual-beam FIB-SEM is used to locate the region of interest with the electron column, before milling around the feature with the ion beam to enable its removal from the bulk with a micromanipulator. By thinning the sample to electron transparency, TEM can be used to determine crystallography and morphology of nanoscale features, dislocation density and type, and local chemistry.

Atom Probe Tomography (APT)

When it is deemed necessary to understand the chemical composition of a nanometre-scale feature, or to determine the compositional change across a microstructural interface, the high compositional and spatial resolution of APT has been shown to be industrially applicable [7].
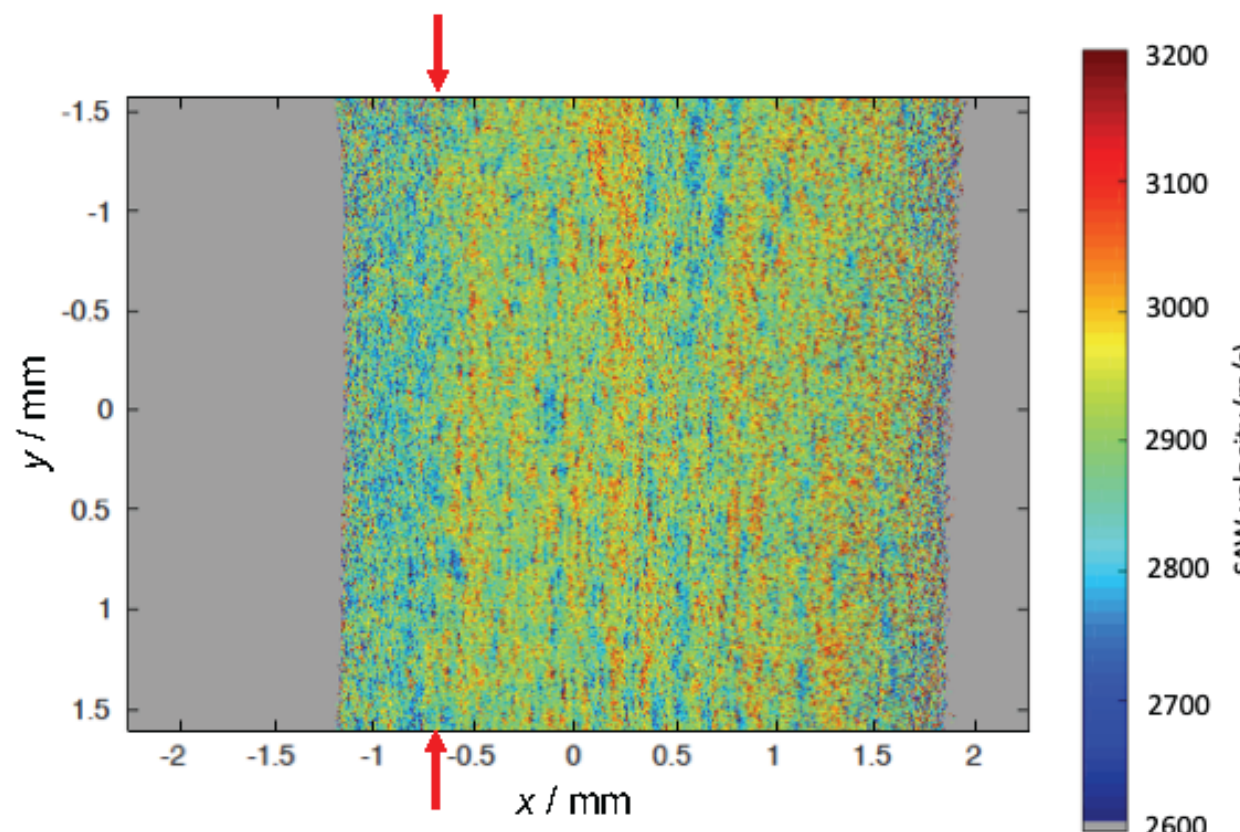

Figure 7: A SRAS velocity map of two Ti-6AI-4V (wt.\%) plates that have been diffusion bonded together (bond line indicated by red arrows). The differences in both grain size (finer in the LHS plate) and texture (macrozones evident in the RHS plate) are evident.

\section{Novel techniques}

Rolls-Royce's work with a number of leading research establishments in the UK aims to develop new techniques and exploit cutting-edge science for industrial applications. 


\section{Micromechanical testing}

Failure investigation currently focusses on characterisation of surfaces and microstructural features to determine the likely root cause of failure. This may seek to infer whether the material's mechanical behaviour at the region of damage initiation was as expected or had been degraded prior to cracking, leading to early failure. Conventional mechanical testing of surrounding material is often insufficiently representative of the region of interest, due to practical difficulties with specimen size and achievable proximity to the feature.

The advent of micro- and mesomechanical testing has made possible the direct measurement of monotonic and fatigue properties across sub-micron to 100 um length scales, Fig. 8 [8]. Complex environmentally degraded surfaces [9] and bulk material can be analysed, as well as fatigue testing of individual or combined microstructural components. This offers a potential step-change in significant investigations that extend to include research activity, as well as more standard usage in future. Another advantage could be testing the effect of non-standard surface conditions such as roughness, residual stress or damage, generally not captured by conventional testing.

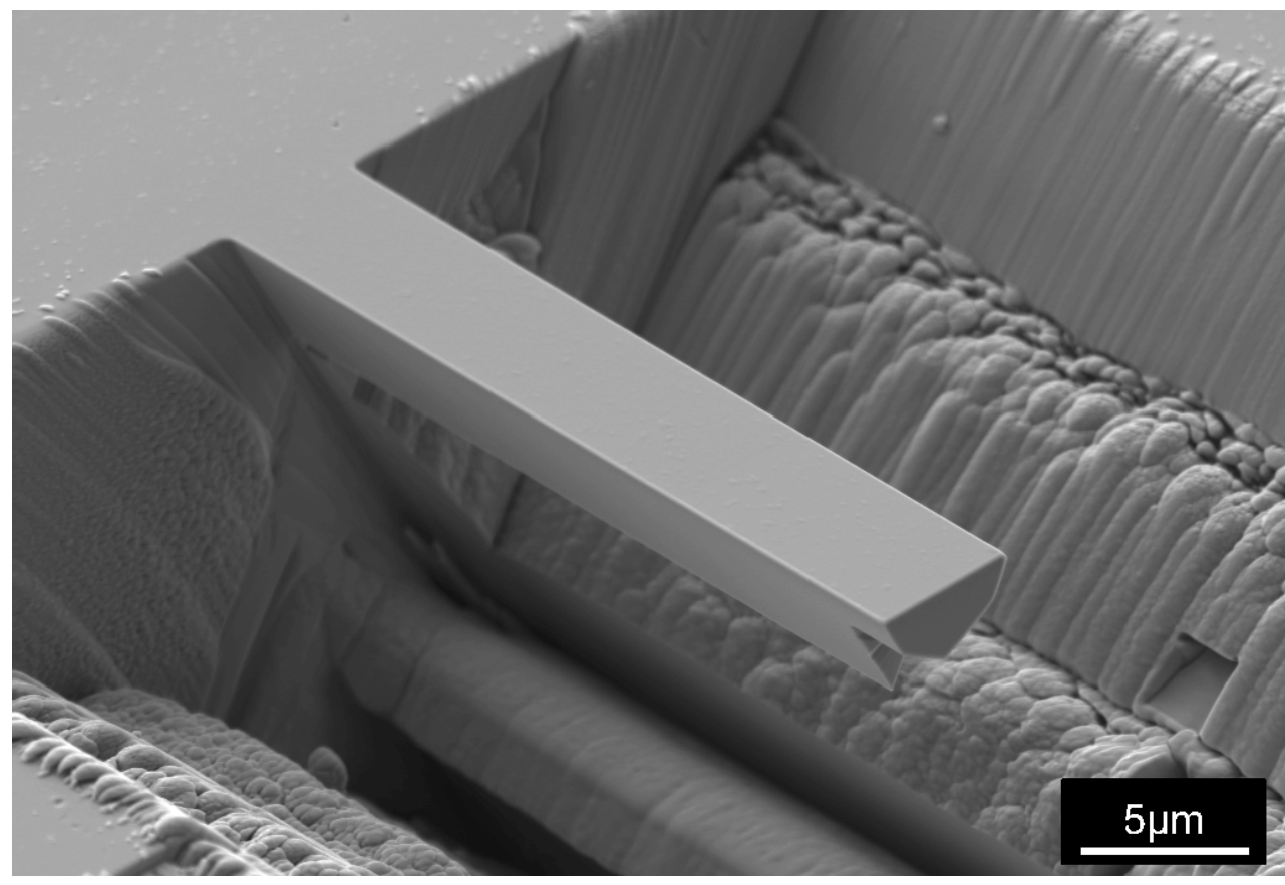

Figure 8: An SEM image of a FIB-milled microcantilever in a titanium alloy, ready for loading with an indenter to investigate the orientation dependence of slip behaviour. In industrial applications, this could be used to probe slip behaviours in specific grains or features of interest on ex-service parts.

High speed atomic force microscopy (HS-AFM)

AFM offers high-resolution 3D measurement of topography, by tracking the deflection of a sharp tip rastered over the surface. Development of AFM towards use in failure investigation could offer insight into sample stress histories. Imaging of fatigue striations has been achieved in research [10], but conventional apparatus is unable to cope with micron-scale height changes commonly seen on fracture surfaces, and is subject to several artefacts and slow scan times. HS-AFM has recently been developed and offers real-time, live imaging across large areas [11]. If challenges with large height ranges can be overcome, HS-AFM may be a useful investigative tool in future.

\section{Micro-computed tomography $(\mu \mathrm{CT})$}

Using $\mu \mathrm{CT}$ to examine cracks in test samples has been shown to be feasible [12], Fig. 9. The transition to using it for ex-service components to identify crack locations and growth paths remains challenging. The complex geometries and sizes of jet engine components cause issues around achieving clear transmission of $X$-rays while maintaining high resolution.

(a)

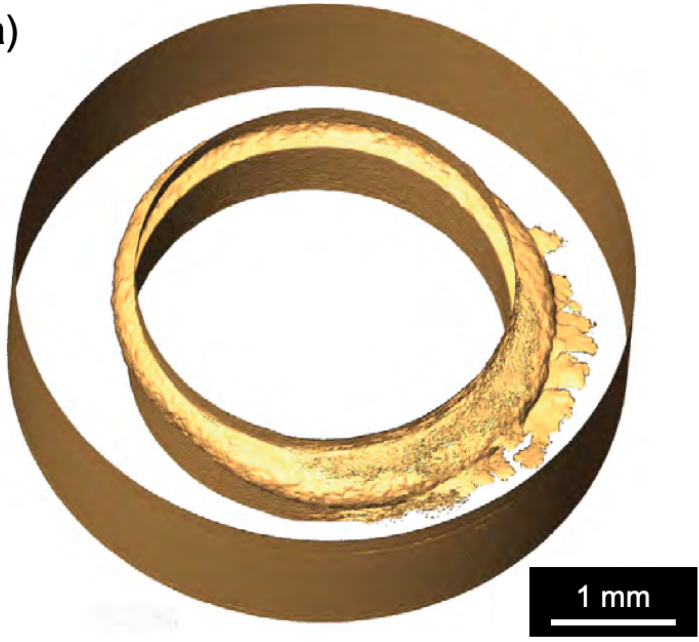

(b)

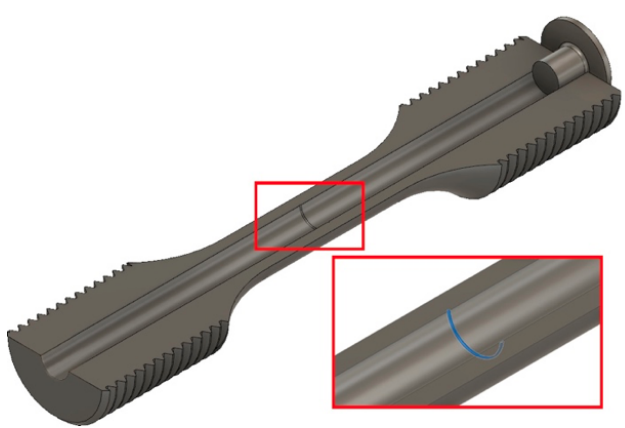

Figure 9: (a) A 3D $\mu \mathrm{CT}$ isosurface reconstruction of a hollow fatigue test specimen, showing a fatigue crack during propagation. (b) The specimen geometry shown in a CAD model. 


\section{Conclusions}

Selecting the right combination of contemporary techniques for failure investigation of an ex-service component allows for much more clarity around the root cause of the failure, alongside deeper understanding of the underlying mechanisms. The field has progressed significantly from a previously limited suite of techniques and continues to develop rapidly. It should also be recognised that, in a commercial environment, there remains a tension between the required level of characterisation of a failed component and the time and cost incurred in performing such analysis.

The importance of strategic development of advanced characterisation techniques for failure investigation cannot be overstated. It is of extreme value to manufacturers to have a wide range of techniques available, ready to be used as and when required. For this reason, Rolls-Royce continues to build and sustain relationships with academia and specialist technique providers to further the development and adaptation of existing and emerging characterisation techniques.

\section{Acknowledgments}

The authors wish to acknowledge the input and contributions of the following colleagues, who have provided access to and insight into the techniques covered in this review: Paul Ashton and Al Cropp (Carl Zeiss UK); Prof. Angus Wilkinson and Dr Jicheng Gong (University of Oxford); Dr Richard Chater and Dr loannis Bantounas (Imperial College London); the team at Loughborough Surface Analysis; Dr Mohammed Azeem (Leicester University); Dr Oliver Payton and Dr Loren Pico (University of Bristol); Dr Richard Smith (University of Nottingham); Jonathan Green and Tomasz Zalewski (Rolls-Royce plc).

\section{References}

[1] https://www.zeiss.com/microscopy/int/products/imaging-systems/smartzoom-5.html (retrieved 2 April 2019).

[2] https://www.zeiss.com/microscopy/int/products/microscope-software.html (retrieved 2 April 2019).

[3] Y. Nukui et al., Mat. Sci. Eng. A 716 (2018) 228-234.

[4] T.P. Chapman et al., Corros. Sci. 96 (2015) 87-101.

[5] https://www.zeiss.com/microscopy/int/products/confocal-microscopes.html (retrieved 2 April 2019).

[6] M. Brown et al., CIRP Journal of Manufacturing Science and Technology 23 (2018) 39-53.

[7] P.A.J. Bagot et al., Scripta Mater. 148 (2018) 24-28.

[8] D. Rugg et al., Mat. Sci. Eng. A 599 (2014) 166-173.

[9] A. Stratulat et al., Corros. Sci. 104 (2016) 9-16.

[10] P.O. Tympel, PhD thesis, Imperial College London, UK (2016).

[11] B.P. Brown et al. Small 9(19) (2013) 3201-3211.

[12] T.P. Chapman et al., Acta Mater. 99 (2015) 49-62. 